\title{
The Influence of Ketogenic Diet on Liver Function in Children and Adolescents with Intractable Epilepsy
}

\author{
Shahabeddin Rezaei, ${ }^{1,2}$ Mona Kavoosi, ${ }^{1}$ Reza Shervin Badv, ${ }^{3}$ Mahmoud Mohammadi, ${ }^{3}$ Gholam Reza \\ Zamani, ${ }^{3}$ Mahmoud Reza Ashrafi, ${ }^{3}$ Mir Saeed Yekaninejad, ${ }^{4}$ and Maryam Mahmoudi ${ }^{1,5,6,}{ }^{*}$ \\ ${ }^{1}$ Department of Cellular Molecular Nutrition, School of Nutritional Sciences and Dietetics, Tehran University of Medical Sciences, Tehran, Iran \\ ${ }^{2}$ Students' Scientific Research Center, Tehran University of Medical Sciences, Tehran, Iran \\ ${ }^{3}$ Paediatrics Centre of Excellence, Department of Paediatric Neurology, Children's Medical Centre, Tehran University of Medical Sciences, Tehran, Iran \\ ${ }^{4}$ Department of Epidemiology and Biostatistics, School of Public Health, Tehran University of Medical Sciences (TUMS), Tehran, Iran \\ ${ }^{5}$ Pediatric Gastroenterology and Hepatology Research Center, Children's Medical Center, Tehran University of Medical Sciences, Tehran, Iran \\ ${ }^{6}$ Dietetics and Nutrition Experts Team (DiNET), Universal Scientific Education and Research Network (USERN), Tehran, Iran \\ "Corresponding author: Maryam Mahmoudi, Associate Professor of Nutrition, Department of Cellular and Molecular Nutrition, School of Nutritional Sciences and Dietetics, \\ Tehran University of Medical Sciences, Tehran, Iran. P.O. Box: 14155-6447. Tel: +98-2188955698, E-mail: m-mahmoudi@sina.tums.ac.ir
}

Received 2017 April 13; Revised 2017 May 04; Accepted 2017 July 23.

\begin{abstract}
Background: High-fat content and carbohydrate restriction of the ketogenic diet (KD) increases the workload of some organs, especially the liver. Moreover, most of the antiepileptic drugs (AEDs) are metabolized in the liver; therefore, the function of the liver could be influenced during the treatment with KD. This study was undertaken to investigate the influence of the ketogenic diet on liver function.

Methods: A prospective, open-label study was carried out. A total of 28 patients with intractable epilepsy initiated the diet. All patients were children and adolescents who did not respond to at least 2 AEDs. Liver function tests including Alanine aminotransferase (ALT), Aspartate aminotransferase (AST), and alkaline phosphatase (ALP) were analyzed before and after 3 months on the KD.

Results: Fourteen patients with the median age of 4.12 (2.31 - 9.70) years completed the study. The serum levels of ALP and AST did not differ significantly before and after 3 months on the diet (P value $>0.05$ ). There was a significant difference in the serum level of ALT before and after 3 months of treatment (13 vrs 20, $\mathrm{P}=0.014$ ).

Conclusions: Albeit, it seems that KD did not have any influence on the serum levels of AST and ALP; however, it had a significant effect on the serum level of ALT. More investigations with a larger sample size and longer follow-up duration are needed to approve abovementioned results.
\end{abstract}

Keywords: Ketogenic Diet, Epilepsy, Alanine Aminotransferase, Aspartate Aminotransferase, Alkaline Phosphatase, Child, Adolescent

\section{Background}

The ketogenic diet (KD) is a low-carbohydrate, high fat, adequate-protein diet. Primarily, KD was introduced to treat intractable epilepsy in 1921 (1), however, in the recent years, use of KD expanded to the other diseases including inherent metabolic diseases (2), autism (3), and glioblastoma (4). The diet initially used was the classical KD, which long chain triglycerides are the primary energy source of the diet (90\%) (5). The diet is calculated based on fat to carbohydrate plus protein ratio (ketogenic ratio). The ratio in the classical ketogenic diet is 4:1 or 3:1, $4 \mathrm{~g}$ (or 3 gram) of fat to $1 \mathrm{~g}$ of combined protein and carbohydrate (5).

Under the $\mathrm{KD}$ treatment, the body mimics the metabolic conditions found during fasting (6). Fat becomes predominant energy sources during KD treatment instead of glucose (6). In the liver, long-chain fatty acids converted to ketone bodies including $\beta$-hydroxybutyrate, acetoacetate, and acetone through the $\beta$-oxidation pathway (6).

Generally, patients with intractable epilepsy need longterm treatment with antiepileptic drugs (AEDs) (7). Most of the AEDs are predominantly metabolized by the liver, including valproate (VPA), carbamazepine, lamotrigine, phenobarbital, oxcarbazepine, phenytoin, felbamate, ethosuximide, and benzodiazepines (8). Additionally, some are AEDs partially metabolized by the liver including levetiracetam, topiramate, and zonisamide (8).

It seems that the combination of the KD and AEDs could influence the liver function. Several studies reported the short- and long-term adverse effects of $\operatorname{KD}(9,10)$. Yet, the influence of $\mathrm{KD}$ on the liver function is ambiguous. Therefore, this study was conducted to investigate the influence of classical KD on the serum levels of liver enzymes in epileptic children and adolescents. 


\section{Methods}

\subsection{Study Population}

A prospective, open-label study design was employed. The present study was carried out at the children's medical centre of Tehran University of Medical Sciences (TUMS) between September 2015 and October 2016. Patients with intractable epilepsy who had the following criteria were included in this study; 1 . aged between 2 and 18 years and 2. did not respond to at least 2 anti-epileptic medications. The exclusion criteria included: 1 . previous treatment with $\mathrm{KD}$; 2 . history of metabolic diseases that have contraindicating use of the ketogenic diet, including pyruvate carboxylase deficiency and medium-chain acyl-CoA dehydrogenase; 3. previous history of liver diseases; 4 . history of dyslipidemia; 5. history of thyroid diseases; 6 . history of renal diseases; and 7. difficulty in the swallowing.

\subsection{Ketogenic Diet Protocol}

Classical KD was initiated for the patients. The ketogenic ratio of 4:1, 4 grams of fat to 1 gram of carbohydrate plus protein, was applied for the patients. No fasting, calorie, or liquid restrictions were implemented. Prior to initiation of the diet, patients were screened for metabolic diseases that have contraindication for KD. Since the KD is nutritionally deficient, the multivitamin and mineral as well as additional calcium plus vitamin D supplements were given to all the patients. All patients were followed up by telephone during the treatment and outpatient visit after 1 and 3 months on the diet.

\subsection{Anthropometric Assessments}

Weight and height of the patients were measured at baseline and 3 months after the initiation of the diet. Prior to the measurement, participants were asked to take off their shoes and heavy clothes. A standardized balancebeam scale was used for measurement the weight $(\mathrm{kg})$. Height $(\mathrm{cm})$ was measured by stadiometer. The body mass index (BMI) was calculated as the measured body weight in $\mathrm{kg}$ divided by the square of the measured height in meters $\left(\mathrm{kg} / \mathrm{m}^{2}\right)$

\subsection{Liver Function Parameters}

Blood sample was obtained in the morning after an overnight fasting. Liver function parameters, namely alanine aminotransferase (ALT), aspartate aminotransferase (AST), and alkaline phosphatase (ALP) were analyzed before initiation of the diet and at month-3.

\subsection{Ethics Consideration}

The study was initiated after approval of the ethical committee of the research deputy of Tehran University of Medical Sciences (ethical code, IR.TUMS.REC.1394.181512.11.94). Informed consent was obtained from all the parents of patients before beginning the diet. Patients participated in the study voluntarily and the parents of the patient were assured of being able to exit the study at any stage in the study. No additional costs were incurred by family of the patients.

\subsection{Statistical Analyses}

A Wilcoxon signed rank test was used to compare the median serum levels of liver function tests before and after commencement of the KD. The results are depicted as median and interquartile range (25th - 75th percentiles). The statistical significance level for all tests was $\mathrm{P}<0.05$. Statistical package for social sciences (SPSS, Chicago, IL, USA) version 16.0 was used for the statistical analysis.

\section{Results}

A total of 28 patients with intractable epilepsy initiated the diet, among them, 14 patients completed the study, including 11 boys. Fourteen patients ceased the diet due to intolerance of the diet, unwillingness of family, minor acidosis, and pneumonia. A median age at initiation of the diet was 4.12 years (Interquartile range: 2.31 - 9.70). Table 1 demonstrated the general characteristics of the patients.

The median (Interquartile range) serum levels of AST, ALT, and ALP at baseline were 21(18-26)U/L,13 (8-21)U/L, and 448 (288 - 708) U/L, respectively. After 3 months, the serum level of AST was 29 (17-38) U/L, the serum level of ALT was 20 $(14$ - 44) U/L, and the serum level of ALP was 453 (298 - 569) $\mathrm{U} / \mathrm{L}$.

There was no difference in the level of ALP at baseline and month-3 $(\mathrm{P}=0.249)$ (Figure 1). Pvalue of 0.108 divulged that the serum level of AST did not differ significantly before and after 3 months of treatment (Figure 2). There was a significant difference in the serum level of ALT before and after 3 months of treatment $(\mathrm{P}=0.014)$ (Figure 3 ).

\section{Discussion}

In the present study, we prospectively investigated the liver function biomarkers of patients with intractable epilepsy. The results of the current study depicted that classical KD did not have any pernicious influence on the serum levels of AST and ALP; however, it has the effect on the serum level of ALT. 
Table 1. General Characteristics of the Study Population

\begin{tabular}{lc}
\hline General Information & No. (\%) \\
\hline Male gender & $11(78.6 \%)$ \\
Female gender & $3(21.4 \%)$ \\
& Median (Interquartile Range) \\
Age $(\mathbf{y})$ & $4.12(2.31-9.70)$ \\
Height $(\mathbf{c m})$ & $102.85(90.00-137.92)$ \\
Weight $(\mathbf{k g})$ & $15.00(11.65-31.25)$ \\
BMI $\left(\mathbf{k g} / \mathbf{m}^{2}\right)$ & $14.75(13.80-16.35)$ \\
\hline
\end{tabular}

Abbreviation: BMI, body mass index.

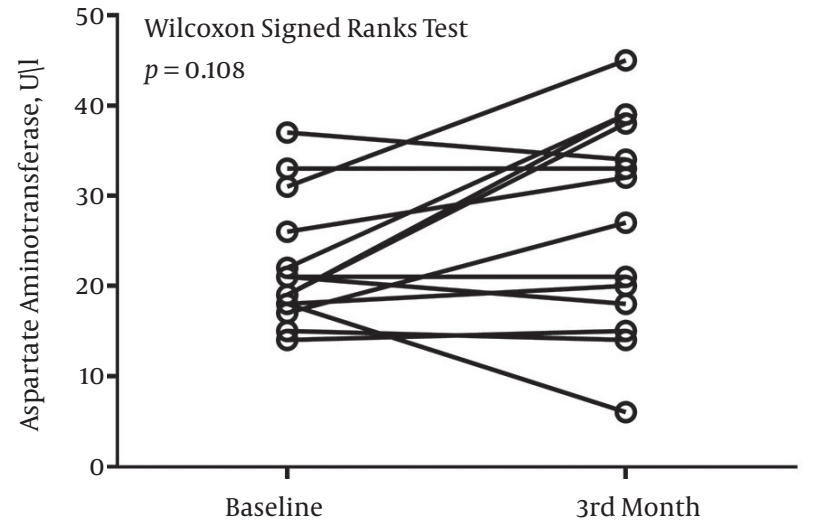

Figure 1. The Serum Levels of Aspartate Aminotransferase Before and After Three Months on the Diet

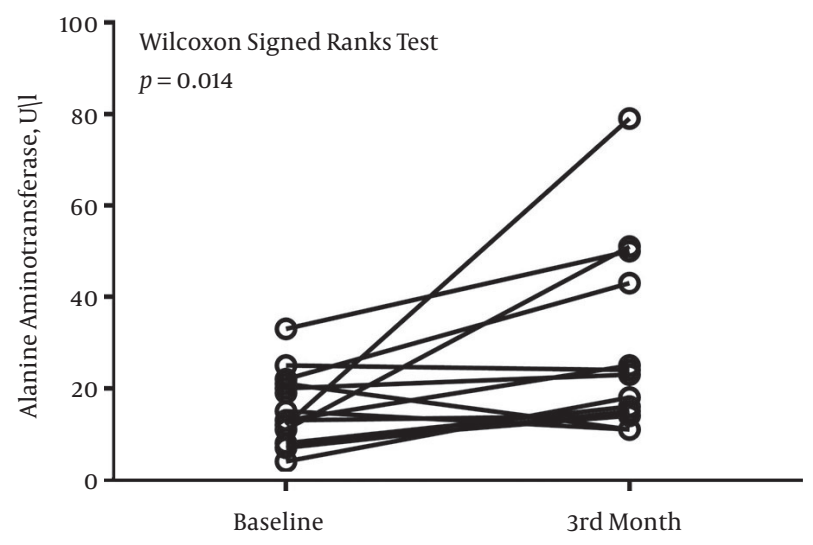

Figure 2. The Serum Levels of Alanine Aminotransferase Before and After Three Months on the Diet

The KD has some short-term and long-term detrimental effects on patients (9). Hypoglycemia, acidosis, nausea,

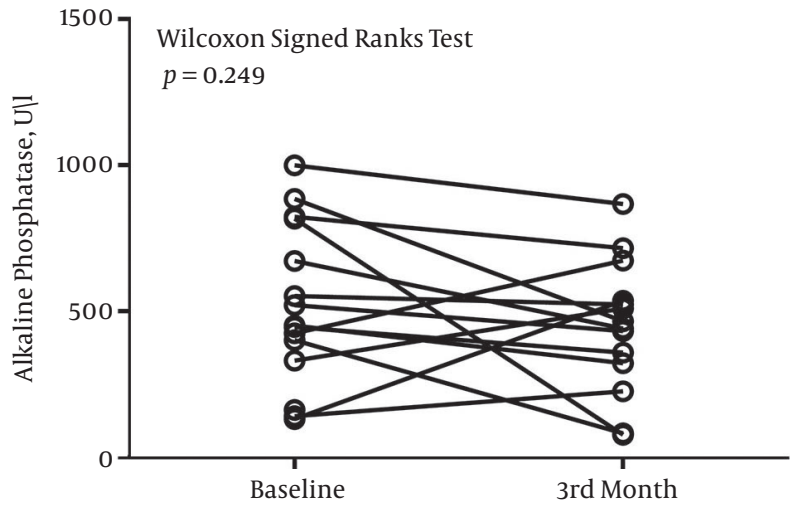

Figure 3. The Serum Levels of Alkaline Phosphatase Before and After Three Months on the Diet

constipation, and vomiting are some examples of shortterm side effects (9). Some of the long-term adverse effects are dyslipidemia, kidney stone, osteopenia, and carnitine deficiency (11). Earlier studies reported several cases where following use of $\mathrm{KD}$, the serum levels of liver enzymes altered $(9,12)$. In the study that was conducted by Arslan et al., they retrospectively evaluated the long-term effect of the KD in patients (12). After 3 months, 2 patients had high serum levels of ALT and AST. One of the patients used VPA as well as levetiracetam and the other took phenobarbital and levetiracetam prior to initiation of the diet (12). Moreover, in a study done by Kang et al, among the 129 patients that were included in the study, 10 of them experienced the elevation of ALT and AST (9).

Previous studies revealed that combination of AEDs and KD, especially VPA, could influence liver function. Stevens et al. reported on an 18-month old girl who experienced hepatic dysfunction following initiation of the KD. The patient took VPA concurrently with the KD. The patient's ALT and AST levels increased dramatically after 1 day 
on KD, from $13 \mathrm{IU} / \mathrm{L}$ to $750 \mathrm{IU} / \mathrm{L}$ and from $28 \mathrm{IU} / \mathrm{L}$ to $785 \mathrm{IU} / \mathrm{L}$, respectively. After ceasing the VPA, the serum levels of ALT and AST became normal (13).

One of our patients had a significant elevation of the serum levels of ALS and AST. The case was 13 years old. He took nitrazepam and VPA. His baseline serum levels of AST and ALT were $19 \mathrm{U} / \mathrm{L}$ and $12 \mathrm{U} / \mathrm{L}$, respectively. After 3 months of treatment, the serum levels of AST and ALT raised to 38 $\mathrm{U} / \mathrm{L}$ and $79 \mathrm{U} / \mathrm{L}$, respectively.

In the study that was done by Lyczkowski et al, 2 patients who took KD and VPA concomitantly experienced raises of the serum liver function enzymes (14). In 1 patient after 3 weeks, the serum levels of ALT and AST were increased to $201 \mathrm{U} / \mathrm{L}$ and $161 \mathrm{U} / \mathrm{L}$, respectively. The 2 nd patient experienced the elevation of liver function tests after 2 weeks on the diet (ALT, 229 U/L; AST, 238 U/L). Albeit, after comparison of the adverse effects of patients who got the combination of KD and VPA and the patients who took exclusively KD, they concluded that concomitant introduction of KD and VPA did not increase the chance of hepatotoxicity (14). In addition, Ballaban-Gil et al. reported 2 cases that had the marked elevation of liver function biomarkers during initiation of the diet and 13 months after commencement of $\mathrm{KD}(15)$.

One of the mechanisms for abnormality of the liver enzymes could be the influence of the diet and AEDs on carnitine storage. Carnitine is an amino acid derivative that is responsible for transport of the long-chain fatty acids to the mitochondria (16). Previous studies unveiled that both AEDs, especially VPA and KD, deplete the storage of carnitine (16). VPA is predominantly metabolized by the liver through glucuronidation and oxidation $(8,16)$. VPA undergoes 2 oxidation pathways namely beta- and omegaoxidation. Conventionally, beta-oxidation dominates over omega-oxidation (17). In the beta-oxidation pathway, VPA combines with carnitine to transport to the mitochondria, therefore, the content of carnitine declined gradually (17). Moreover, high amounts of long-chain triglyceride decrease carnitine level. In fact, adding KD to some AEDs, particularly VPA, has the synergistic influence on depletion of carnitine storage (16).

Decreasing the amount of carnitine leads to hepatotoxicity (16). The precise mechanism is not clear, however, earlier studies proposed that carnitine reduction leads to an imbalance between gamma- and beta-oxidation. Through gamma-oxidation pathway, some toxic metabolites including 4-en-VPA are produced. In the absence of carnitine, VPA undergoes to gamma-oxidation instead of the betapathway, therefore, accumulation of toxic metabolites leads to hepatotoxicity $(16,18)$.

This study had some limitations. One of the limitations of the present study was the number of sample size. The absence of the control group was another limitation of the current study. In conclusion, it seems that KD did not have any influence on the serum levels of AST and ALP; however, it had the effect on the serum level of ALT.

\section{Acknowledgments}

This study was supported by Tehran University of Medical Sciences; grant no. 94-03-161-30342.

\section{References}

1. Wilder R. The effects of ketonemia on the course of epilepsy. Mayo Clin Proc. 1921.

2. Scholl-Burgi S, Holler A, Pichler K, Michel M, Haberlandt E, Karall D. Ketogenic diets in patients with inherited metabolic disorders. JInherit Metab Dis. 2015;38(4):765-73. doi:10.1007/s10545-015-9872-2. [PubMed: 26109259].

3. Castro. K. , Faccioli LS, Baronio D, Gottfried C, Perry IS, dos Santos Riesgo R. Effect of a ketogenic diet on autism spectrum disorder: A systematic review. Res Autism Spectr Disord. 2015;20:31-8.

4. Winter SF, Loebel F, Dietrich J. Role of ketogenic metabolic therapy in malignant glioma: A systematic review. Crit Rev Oncol Hematol. 2017;112:41-58. doi: 10.1016/j.critrevonc.2017.02.016. [PubMed: 28325264].

5. Winesett SP, Bessone SK, Kossoff EH. The ketogenic diet in pharmacoresistant childhood epilepsy. Expert Rev Neurother. 2015;15(6):621-8. doi: 10.1586/14737175.2015.1044982. [PubMed: 25994046].

6. Masino SA, Rho JM. Mechanisms of Ketogenic Diet Action. In: Noebels JL, Avoli M, Rogawski MA, Olsen RW, Delgado-Escueta AV, editors. Jasper's Basic Mechanisms of the Epilepsies. 4th ed. ; 2012.

7. McCorry D, Chadwick D, Marson A. Current drug treatment of epilepsy in adults. Lancet Neurol. 2004;3(12):729-35. doi:10.1016/S14744422(04)00935-4. [PubMed: 15556805].

8. Ahmed SN, Siddiqi ZA. Antiepileptic drugs and liver disease. Seizure. 2006;15(3):156-64. doi: 10.1016/j.seizure.2005.12.009. [PubMed: 16442314].

9. Kang HC, Chung DE, Kim DW, Kim HD. Early- and late-onset complications of the ketogenic diet for intractable epilepsy. Epilepsia. 2004;45(9):1116-23. doi: 10.1111/j.0013-9580.2004.10004.x. [PubMed 15329077].

10. Lin A, Turner Z, Doerrer SC, Stanfield A, Kossoff EH. Complications During Ketogenic Diet Initiation: Prevalence, Treatment, and Influence on Seizure Outcomes. Pediatr Neurol. 2017;68:35-9. doi: 10.1016/j.pediatrneurol.2017.01.007. [PubMed: 28188074].

11. Cervenka MC, Henry BJ, Kossoff EH, Zahava Turner R. The ketogenic and modified atkins diets: Treatments for epilepsy and other disorders. Springer Publishing Company; 2016. p. 376.

12. Arslan N, Guzel O, Kose E, Yilmaz U, Kuyum P, Aksoy B, et al. Is ketogenic diet treatment hepatotoxic for children with intractable epilepsy? Seizure. 2016;43:32-8. doi: 10.1016/j.seizure.2016.10.024. [PubMed: 27866088].

13. Stevens CE, Turner Z, Kossoff EH. Hepatic Dysfunction as a Complication of Combined Valproate and Ketogenic Diet. Pediatr Neurol. 2016;54:82-4. doi: 10.1016/j.pediatrneurol.2015.10.006. [PubMed: 26706483].

14. Lyczkowski DA, Pfeifer HH, Ghosh S, Thiele EA. Safety and tolerability of the ketogenic diet in pediatric epilepsy: effects of valproate combination therapy. Epilepsia. 2005;46(9):1533-8. doi: 10.1111/j.15281167.2005.22705.x. [PubMed: 16146450]. 
15. Ballaban-Gil K, Callahan C, O’Dell C, Pappo M, Moshe S, Shinnar S. Com plications of the ketogenic diet. Epilepsia. 1998;39(7):744-8. [PubMed: 9670903].

16. Lheureux PE, Hantson P. Carnitine in the treatment of valproic acid-induced toxicity. Clin Toxicol (Phila). 2009;47(2):101-11. doi: 10.1080/15563650902752376. [PubMed: 19280426].
17. Singh G. The Treatment of Epilepsy. Wiley-Blackwell; 2009. pp. 25972.Management of medical comorbidity associated with epilepsy.

18. Bjornsson E. Hepatotoxicity associated with antiepileptic drugs. Acta Neurol Scand. 2008;118(5):281-90. doi: 10.1111/j.16000404.2008.01009.x. [PubMed: 18341684]. 